Acta Crystallographica Section F

Structural Biology

Communications

ISSN 2053-230X

Greg Mann, ' Jesko Koehnke,

Andrew F. Bent, ${ }^{a}$ Rachael

Graham, ${ }^{\text {a }}$ Wael Houssen, ${ }^{\text {b,c,d }}$

Marcel Jaspars, ${ }^{b}$ Uli Schwarz-

Linek $^{\mathrm{a}}$ and James H. Naismith ${ }^{\mathrm{a} *}$

aBiomedical Sciences Research Complex, University of St Andrews, North Haugh, St Andrews, Fife KY16 8ST, Scotland, 'b Marine Biodiscovery Centre, Department of Chemistry, University of Aberdeen, Meston Walk, Aberdeen AB24 3UE, Scotland, "Institute of Medical Sciences, University of Aberdeen, Aberdeen AB25 2ZD, Scotland, and

dPharmacognosy Department, Faculty of Pharmacy, Mansoura University,

Mansoura 35116, Egypt

Correspondence e-mail: jhn@st-andrews.ac.uk

Received 5 September 2014

Accepted 3 November 2014

PDB reference: PatG-DUF, 4uvq

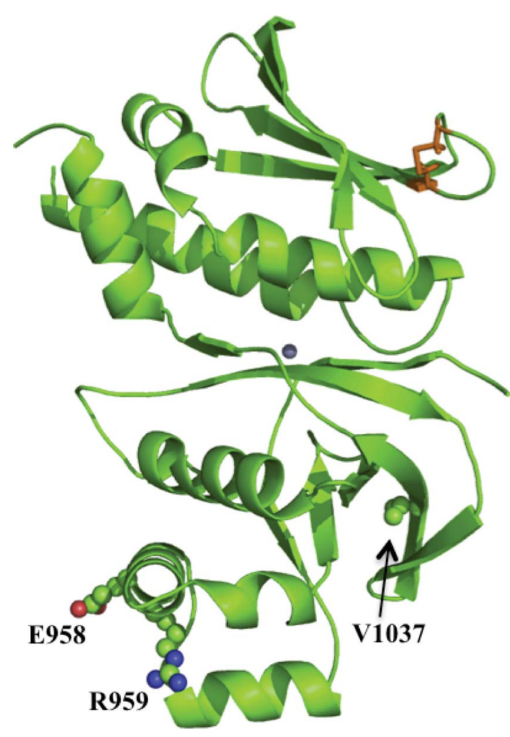

\section{The structure of the cyanobactin domain of unknown function from PatG in the patellamide gene cluster}

Patellamides are members of the cyanobactin family of ribosomally synthesized and post-translationally modified cyclic peptide natural products, many of which, including some patellamides, are biologically active. A detailed mechanistic understanding of the biosynthetic pathway would enable the construction of a biotechnological 'toolkit' to make novel analogues of patellamides that are not found in nature. All but two of the protein domains involved in patellamide biosynthesis have been characterized. The two domains of unknown function (DUFs) are homologous to each other and are found at the C-termini of the multi-domain proteins PatA and PatG. The domain sequence is found in all cyanobactin-biosynthetic pathways characterized to date, implying a functional role in cyanobactin biosynthesis. Here, the crystal structure of the PatG DUF domain is reported and its binding interactions with plausible substrates are investigated.

\section{Introduction}

Prokaryotic secondary metabolism creates an array of bioactive molecules, some of which can be exploited for pharmaceutical development. Cyanobactins, secondary metabolites of cyanobacteria, are a superfamily of ribosomally synthesized and post-translationally modified peptides (RiPPs) that are highly variable with a wide range of bioactivities (Sivonen et al., 2010). Patellamides, eight-residue cyclic peptides that contain oxazoline, thiazoline and thiazole heterocycles and D-amino acids (Schmidt et al., 2005), belong to this superfamily and have attracted considerable interest owing to the ability of patellamide $\mathrm{D}$ to reverse the effects of multidrug resistance in human leukaemia cells (Williams \& Jacobs, 1993; Houssen \& Jaspars, 2010). These natural products are too structurally complex for cost-effective de novo chemical synthesis, and this prevents the creation of novel and diverse analogues with desired properties. Manipulation of the natural product's biosynthetic pathway can offer an alternative, more cost-effective, route to such analogues, but does require a comprehensive understanding of the individual enzymes involved. Moreover, the use of the isolated enzymes can allow the introduction of non-natural functionalities.

Patellamides originate from a longer precursor peptide, which is modified by a series of post-translational modifying (PTM) enzymes to yield the final cyclic product. Their biosynthesis can be attributed to a single operon (patA-patG) encoding the precursor peptide (PatE) and the enzymes required for processing (PatA, PatD and PatG; Schmidt et al., 2005; Fig. 1). The precursor peptide includes a core peptide (or multiple core peptides) that becomes the natural product, as well as conserved recognition sequences and an $\mathrm{N}$-terminal leader peptide, which are discarded during maturation but are required to direct post-translational tailoring. It is assumed, but has not been proven, that all of the chemical tailoring steps are required for biological activity. All but one of the chemical transformations for the tailoring steps have been assigned to domains 


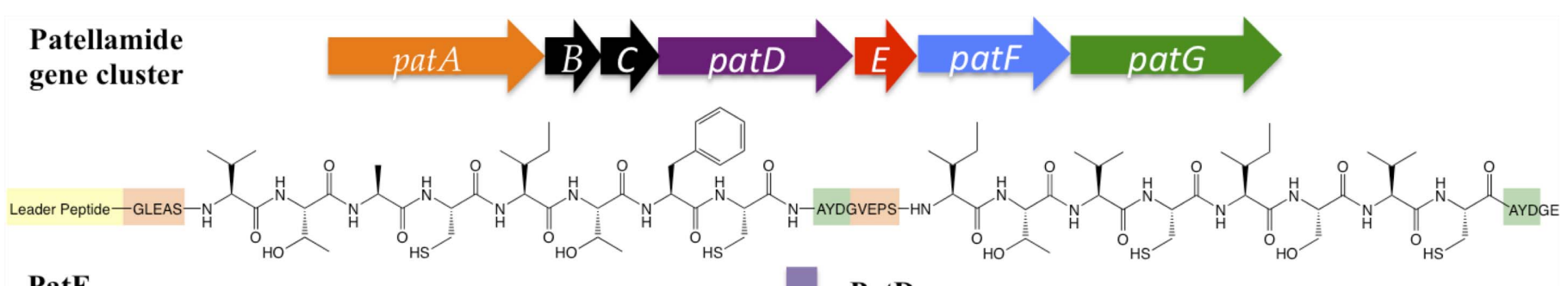

PatE

PatD

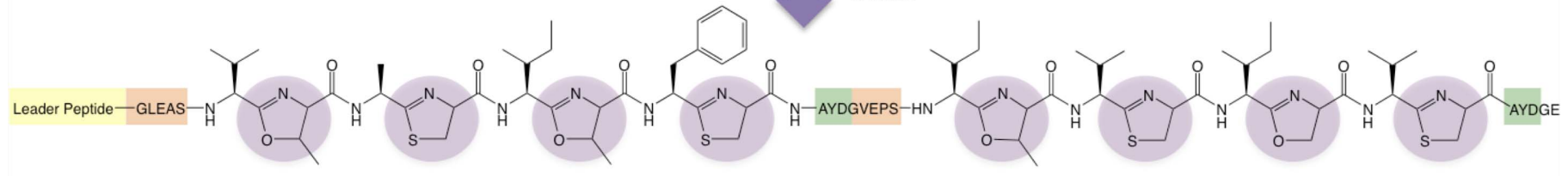

PatE (modified)

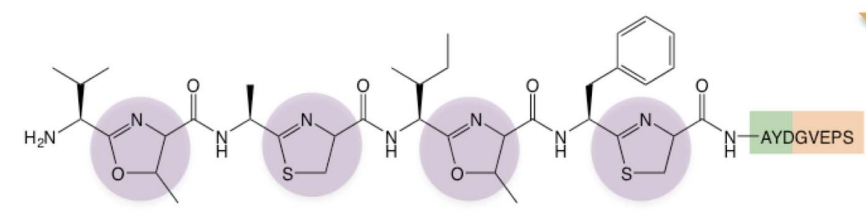

PatE (modified)

Patellamide C

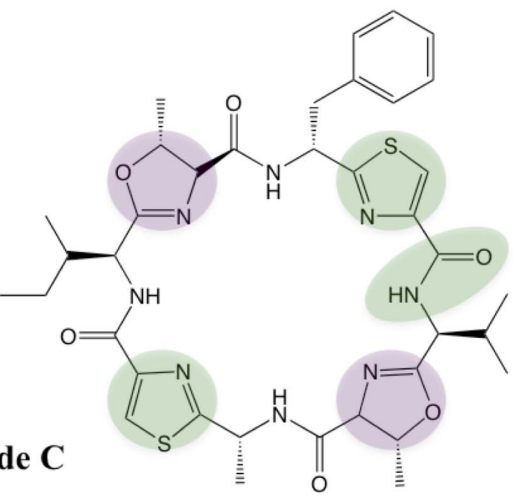

PatA

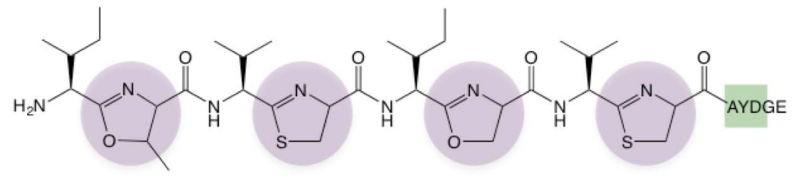

PatG

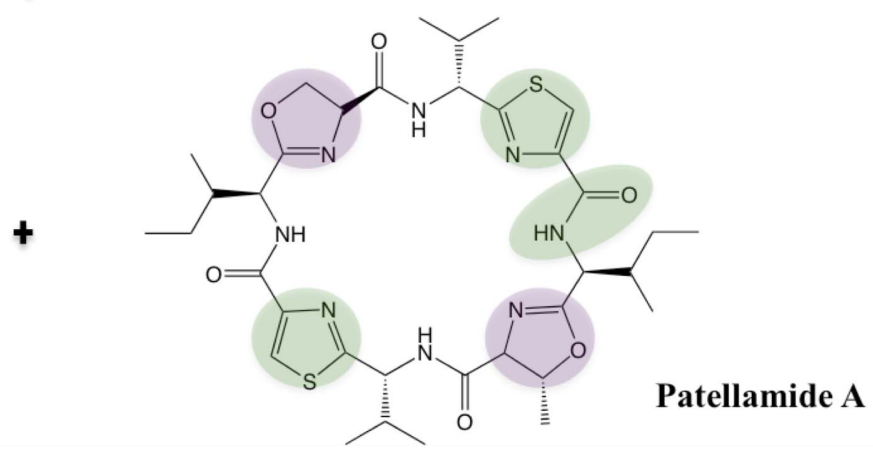

Figure 1

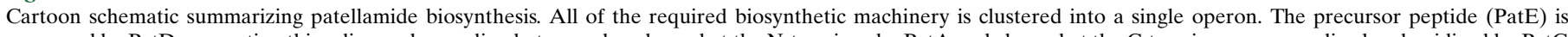

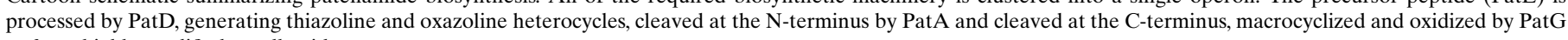
to form highly modified patellamides.

within the processing enzymes (Agarwal et al., 2012; Schmidt et al., 2005). The first step is the heterocyclization of PatE core peptide cysteines, serines and threonines by PatD. This is followed by removal of the N-terminal recognition elements and leader peptide by the protease domain of PatA. Finally, PatG catalyzes the macrocyclization of the modified core peptide, simultaneously removing the C-terminal recognition sequence. Additionally, PatG is responsible for the oxidation of thiazolines to thiazoles (Fig. 1). Structural and mechanistic insights have been reported for the protease domain of PatA (Houssen et al., 2012; Agarwal et al., 2012), the macrocyclization domain of PatG (Koehnke et al., 2012; Agarwal et al., 2012), the heterocyclase TruD (Koehnke et al., 2013; a close homologue of PatD from the trunkamide pathway) and the apparently inactive prenyl transferase PatF (Bent et al., 2013). Only epimerization (which is likely to precede thiazoline oxidation) is unassigned to a protein domain, but it has been suggested that this could be chemically spontaneous (Milne et al., 2006). Whether the epimerization and oxidation occur in the linear or the macrocyclic form is unknown. Two gene products, PatB and PatC, have been reported as non-essential to synthesis, and their role is unknown (Schmidt et al., 2005).

PatA and PatG both contain C-terminal domains of unknown function (DUFs) and these domains are homologous (56\% identical) to each other (Fig. 2a). The gene encoding PatG-DUF (amino-acid residues 914-1191) was amplified from gDNA isolated from Prochloron sp. that were isolated from Pacific reef samples. The exact strain could not be determined and the amino-acid sequence of PatGDUF (PatG-DUF sp.) differs at three points (E958D, R959Y and V1037M) from the deposited amino-acid sequence of PatG-DUF isolated from $P$. didemni (PatG-DUF ${ }_{d i}$; accession No. AAY21556.1; Fig. $2 b)$. It is unclear as to whether these differences arose as a result of natural variance amongst species or owing to a cloning artefact.

The DUF domain is conserved in all patellamide-like cyanobactin biosynthetic pathways characterized to date (Supplementary Fig. $\mathrm{S}^{\mathbf{1}}$ ), suggesting it may play a functional role. To gain some insight into the DUF domain, we have determined the crystal structure of PatG-DUF sp. $_{\text {. }}$ to a resolution of $1.72 \AA$, and using isothermal titration calorimetry (ITC) and nuclear magnetic resonance spectroscopy (NMR) we have probed its binding to some plausible substrates: the precursor peptide and intermediates formed during biosynthesis (Fig. 1).

\footnotetext{
${ }^{1}$ Supporting information has been deposited in the IUCr electronic archive (Reference: SX5117).
} 


\section{Methods}

2.1. Expression of native and SeMet PatG-DUF

The C-terminal domain of PatG from Prochloron sp. (PatGDUF $_{\text {sp. }}$; amino-acid residues 914-1191) was cloned from full-length pat $G$ into the pHISTEV plasmid with an $\mathrm{N}$-terminal $\mathrm{His}_{6}$ tag and a Tobacco etch virus (TEV) protease site (Liu \& Naismith, 2009). The protein was expressed in Escherichia coli BL21 (DE3) cells grown in Luria-Bertani (LB) medium supplemented with $50 \mu \mathrm{g} \mathrm{ml}^{-1}$ kanamycin. Cultures were grown at $37^{\circ} \mathrm{C}$ and $200 \mathrm{rev} \mathrm{min}^{-1}$ until the $\mathrm{OD}_{600}$ reached 0.6 ; the cells were then induced by the addition of isopropyl $\beta$-D-1-thiogalactopyranoside (IPTG; final concentration $1 \mathrm{mM}$ ) and further grown at $18^{\circ} \mathrm{C}$ and $200 \mathrm{rev} \mathrm{min}^{-1}$ overnight.

L-Selenomethionine-labelled (SeMet) PatG-DUF sp. $_{\text {. was expressed }}$ from $E$. coli BL21 (DE3) cells grown in minimal medium supplemented with glucose-free nutrient mix, $50 \mu \mathrm{g} \mathrm{ml}^{-1}$ kanamycin and $5 \%$ glycerol. This medium was inoculated with an overnight culture grown in LB, which was washed three times in minimal medium. After $15 \mathrm{~min}$ growth at $37^{\circ} \mathrm{C}, 60 \mathrm{mg} \mathrm{l}^{-1}$ L-selenomethionine was added. The cultures were returned to $37^{\circ} \mathrm{C}$ and $200 \mathrm{rev} \mathrm{min}^{-1}$ and grown until the $\mathrm{OD}_{600}$ reached 0.6 , when $100 \mathrm{mg} \mathrm{l}^{-1}$ lysine, phenylalanine, threonine and $50 \mathrm{mg} \mathrm{l}^{-1}$ isoleucine and valine were added. After incubation for a further $20 \mathrm{~min}$, expression was induced with IPTG (final concentration $1 \mathrm{mM}$ ) and the cells were grown at $18^{\circ} \mathrm{C}$ for $24 \mathrm{~h}$. For both native and SeMet variants, the cells were harvested by centrifugation $\left(4000 \mathrm{~g}, 4^{\circ} \mathrm{C}, 15 \mathrm{~min}\right)$.

\subsection{Purification of native and SeMet PatG-DUF}

Cell pellets were resuspended in lysis buffer [20 $\mathrm{m} M$ bis-tris $\mathrm{pH} 6.8$, $150 \mathrm{~m} M \mathrm{NaCl}, 20 \mathrm{~m} M$ imidazole, $3 \mathrm{~m} M \beta$-mercaptoethanol (BME), $0.1 \%(v / v)$ Triton X-100] supplemented with $0.4 \mathrm{mg}$ DNAse per gram of wet cell pellets and cOmplete EDTA-free protease-inhibitor tablets (Roche; one tablet per $50 \mathrm{ml}$ resuspension). The cells were lysed via passage through a cell disruptor at $207 \mathrm{MPa}$ (Constant Systems) and the cell debris was removed by centrifugation $(40000 \mathrm{~g}$, $4{ }^{\circ} \mathrm{C}, 20 \mathrm{~min}$ ). The supernatant was loaded onto a pre-equilibrated $\mathrm{Ni}$-NTA column at $4^{\circ} \mathrm{C}$ and the column was washed with 80 column volumes of lysis buffer. The protein was eluted from the column in elution buffer [20 $\mathrm{m} M$ bis-tris $\mathrm{pH} 6.8,150 \mathrm{~m} M \mathrm{NaCl}, 250 \mathrm{~m} M$ imidazole, $3 \mathrm{mM}$ BME, $0.1 \%(v / v)$ Triton $\mathrm{X}-100$ ] and fractions containing protein were pooled and passed over a desalting column (Desalt 16/10, GE Healthcare) to exchange them into 'low imidazole' buffer $(20 \mathrm{~m} M$ Tris- $\mathrm{HCl} \mathrm{pH} 8.0,150 \mathrm{mM} \mathrm{NaCl}, 20 \mathrm{~m} M$ imidazole, $3 \mathrm{~m} M \mathrm{BME}, 10 \%$ glycerol). TEV protease was added at a mass:mass ratio of $1 \mathrm{mg}$ of TEV to $10 \mathrm{mg}$ of protein and the sample was incubated for $2 \mathrm{~h}$ at $20^{\circ} \mathrm{C}$ to remove the $\mathrm{N}$-terminal $\mathrm{His}_{6}$ tag. The digested sample was passed over a second Ni-NTA column and the flowthrough was collected and loaded onto a Superdex 75 gel-filtration column (GE Healthcare) equilibrated in buffer (10 $\mathrm{m} M$ HEPES pH 7.4, $150 \mathrm{~m} M \mathrm{NaCl}, 1 \mathrm{~m} M$ TCEP, $10 \%$ glycerol). The purity of the protein was determined by SDS-PAGE and its integrity was determined by mass spectrometry. Analysis of the mass spectrum of SeMet

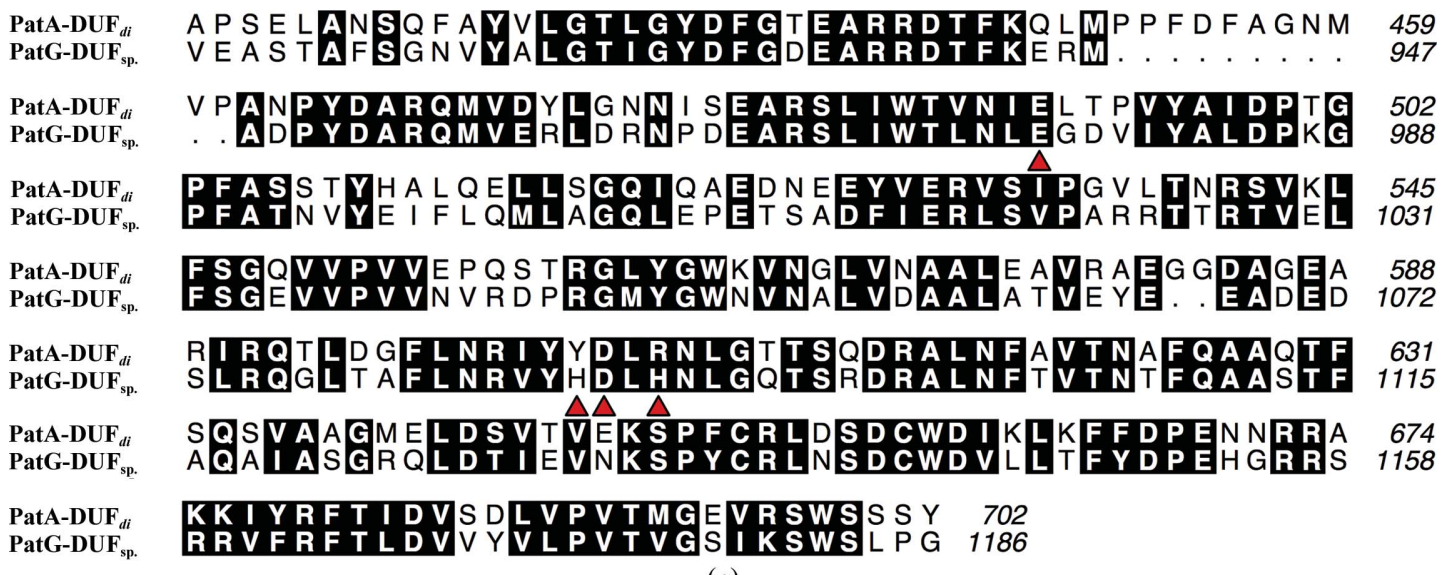

(a)

PatG-DUF PatG-DUF $_{d i}$

PatG-DUF PatG-DUF $_{d i}$

PatG-DUF PatG-DUF $_{d i}$

PatG-DUF sp. PatG-DUF $d i$

PatG-DUF $_{\text {sp }}$ PatG-DUF $_{d i}$

PatG-DUF PatG-DUF $d i$

PatG-DUF PatG-DUF $_{d i}$
VEASTAFSGNVYALGTI GYDFGDEARRDTFKERMADPYD 952 VEASTAFSGNVYALGTIGYDFGDEARRDTFKERMADPYD 952

ARQMVERLDRNPDEARS L I WT L N L E D V I Y A L D K R P A 991 ARQMVDYLDRNPDEARSL I WTLNLEGDV I YALDPKGPFA 991

TNVYEI FLQMLAGQLEPETSADF I ERLSVPARRT TRTVE 1030 TNVYEIFLQMLAGQLEPETSADF I ERLSVPARRT TRTVE 1030

LF SGEVVPVVNVRDPRGMYGWNVNALVDAALATVEYEEA 1069 LF SGE VMP V VN VRDPRGMYGWN VNA L V D A L A T VE YE A 1069

DEDSLRQGLTAF L NRVYHDLHNLGQTSRDRALNF T V T T 1108 DEDSLRQGLTAF LNRVYHDLHNLGQTSRDRA L N T T T N T 1108

FQA A S T F A I A S GRQLDT I E V N K S P Y CR L N S D CWDVL L 1147 FQAASTFAQA I A S RQLD T I V N K S Y CRLNSDCWD VLL 1147 TFYDPEHGRRSRRVFRF T L D V Y V L P T V G I K SWSLPG 1186
TFYDPEHGRRSRRVFRF T L D V V Y V L P V T V G S I K SWSLPG 1186

Figure 2

(b)

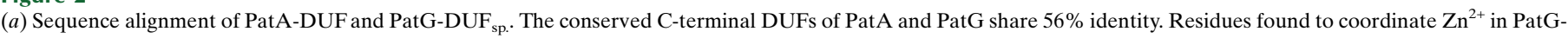

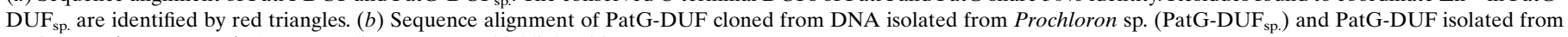
$P$. didemni $\left(\right.$ PatG-DUF ${ }_{d i}$ ). Sequence differences are highlighted in green. 


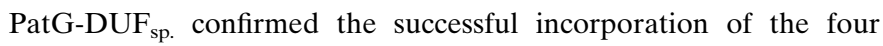
expected SeMet residues. Native and SeMet PatG-DUF $F_{\text {sp. were }}$ concentrated to $4.75 \mathrm{mg} \mathrm{ml}^{-1}$ for crystallography.

\subsection{Mutagenesis}

Mutagenesis of PatG-DUF $F_{\text {sp. }}$ was performed using established protocols (Liu \& Naismith, 2008).

\subsection{CD spectroscopy}

Near-UV CD spectra were recorded on a Jasco J-810 spectropolarimeter with samples in $10 \mathrm{~m} M$ HEPES pH 7.4, $150 \mathrm{~m} M \mathrm{NaCl}$, $1 \mathrm{~m} M$ TCEP, $10 \%$ glycerol.

\subsection{Crystallography}

Crystal screens were set up with the sitting-drop vapour-diffusion method using a Gryphon robot (Art Robbins). The protein was screened against sparse-matrix screens composed of a range of known crystallization conditions at 20 and $4^{\circ} \mathrm{C}$ (Jancarik \& Kim, 1991). Diffraction-quality crystals were grown in a condition consisting of $0.04 \mathrm{M}$ potassium phosphate, 16\%(w/v) PEG 8000, $20 \%(v / v)$ glycerol. Crystals of SeMet PatG-DUF ${ }_{\text {sp. }}$ grew using the same condition as the native protein. A single SeMet PatG-DUF sp. $_{\text {. }}$ crystal was cryoprotected in mother liquor and flash-cooled in liquid nitrogen. A single-wavelength anomalous dispersion (SAD) data set was collected at the Se $K$ absorption edge at $100 \mathrm{~K}$ on beamline I02 at Diamond Light Source. The structure was solved using AutoSol and the chains were built into electron density using AutoBuild from the PHENIX crystallography suite (Adams et al., 2010). Examination of the identified anomalous scattering atoms revealed the Se atoms of the four methionine residues as expected, but also an additional atom at the interface between one monomer and its symmetry mate. The atom was coordinated by two histidine residues, an aspartic acid and a glutamic acid. Based on the anomalous scattering and coordination, we assign this atom as a $\mathrm{Zn}^{2+}$ ion. A map using the phases calculated from the anomalous scattering atoms and a map after density modification of these phases are shown in Supplementary Fig. S2. The structure was manually rebuilt in Coot (Emsley \& Cowtan, 2004) and refined using REFMAC5 (Murshudov et al., 2011). TLS restraints were generated for refinement using the TLSMD server (Painter \& Merritt, 2006) and the structure was validated using MolProbity (Chen et al., 2010). The $\mathrm{Zn}^{2+}$ ion refined with a temperature factor similar to those of the ligating atoms and no additional $F_{\mathrm{o}}-F_{\mathrm{c}}$ density was observed at the site, supporting our identification of the atom as a $\mathrm{Zn}^{2+}$ ion. Full structural statistics are given in Table 1.

All sequence alignments were created using Clustal Omega (Sievers et al., 2014) and presented using ALINE (Bond \& Schüttelkopf, 2009). All structural images were generated using $P y M O L$ (DeLano, 2002).

\subsection{Expression and purification of ${ }^{15} \mathrm{~N}$ PatE and PatD}

For biochemical characterization an engineered PatE variant was used denoted PatE'. PatE' harbours a single core peptide with the sequence ITACITFC (corresponding to the natural product patellamide D) and a C-terminal $\mathrm{His}_{6}$ tag. PatE' and ${ }^{15} \mathrm{~N}$-labelled $\mathrm{PatE}^{\prime}$ were produced as described previously (Koehnke et al., 2013). The enzyme PatD was purified in the same way as previously described for its homologue TruD (Koehnke et al., 2013).

\subsection{Heterocyclization of PatE with PatD}

${ }^{15} \mathrm{~N}$ PatE $(100 \mu M)$ was incubated with PatD $(5 \mu M)$ in PatE' gel-filtration buffer (10 m $M$ HEPES pH 7.4, $150 \mathrm{~m} M \mathrm{NaCl}, 1 \mathrm{~m} M$ TCEP) and the product was purified using size-exclusion chromatography as described previously (Koehnke et al., 2013). The reaction was monitored using MALDI-TOF MS as described previously (Koehnke et al., 2013).

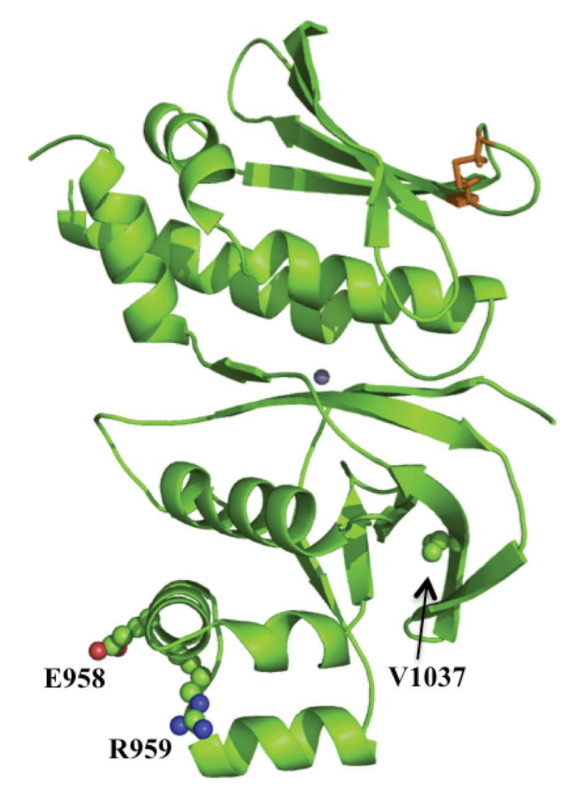

Figure 3

(a)

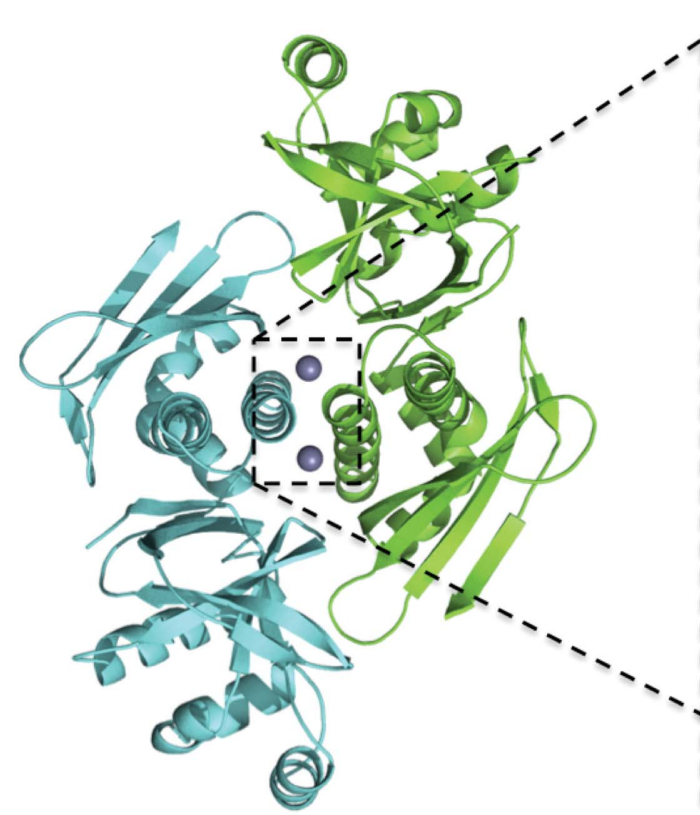

(b)

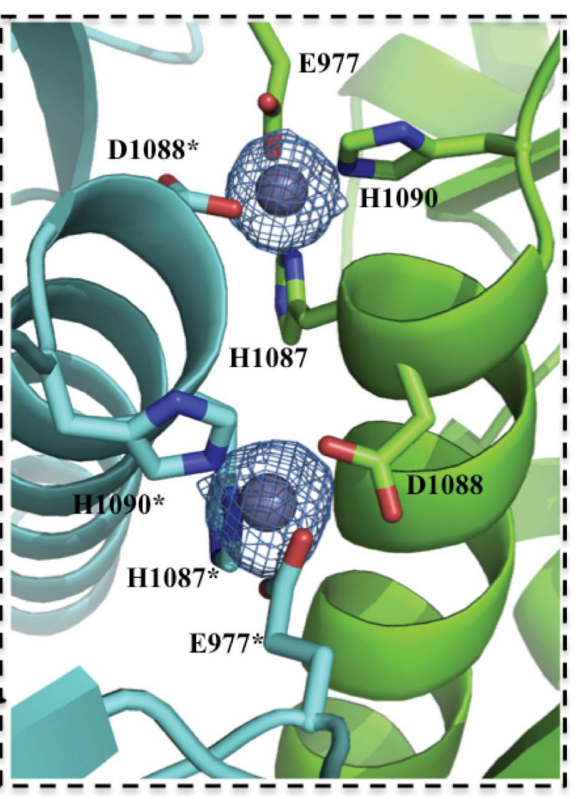

(c)

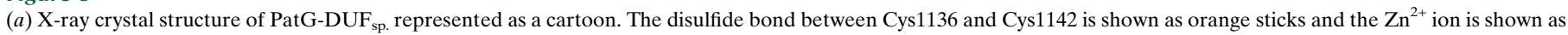

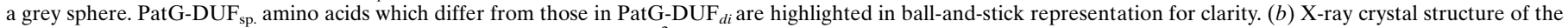

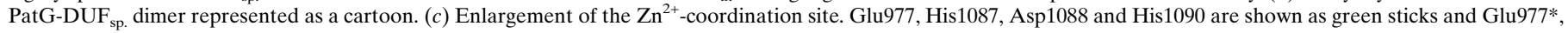

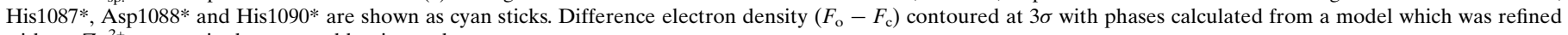
without $\mathrm{Zn}^{2+}$ present is shown as a blue isomesh. 
Table 1

Data-collection and refinement statistics for SeMet PatG-DUF sp. $_{\text {. }}$

\begin{tabular}{|c|c|}
\hline \multicolumn{2}{|c|}{$\begin{array}{l}\text { Anomalous data were collected using a single crystal on beamline I } 02 \text { at the Diamond } \\
\text { Light Source. Statistics are average values; values for the highest resolution shell are } \\
\text { given in parentheses. }\end{array}$} \\
\hline \multicolumn{2}{|l|}{ Data collection } \\
\hline Wavelength $(\AA)$ & 0.9797 \\
\hline Space group & $P 2_{1} 2_{1} 2$ \\
\hline Unit-cell parameters $\left(\AA,{ }^{\circ}\right)$ & $\begin{array}{c}a=64.1, b=96.2, c=40.2, \\
\alpha=\beta=\gamma=90.0\end{array}$ \\
\hline Resolution ( & $53.17-1.72(1.77-1.72)$ \\
\hline$\langle I / \sigma(I)\rangle$ & $12.1(3.4)$ \\
\hline$R_{\text {merge }}(\%)$ & $14.5(89.6)$ \\
\hline Completeness (\%) & $99.9(100)$ \\
\hline Multiplicity & $14.0(11.3)$ \\
\hline Anomalous completeness (\%) & $99.9(99.9)$ \\
\hline Anomalous multiplicity & $7.3(5.7)$ \\
\hline Initial map correlation & 0.4 \\
\hline \multicolumn{2}{|l|}{ Refinement } \\
\hline$R$ factor $(\%)$ & $17.5(23.9)$ \\
\hline$R_{\text {free }}(\%)$ & $20.0(29.5)$ \\
\hline R.m.s.d., bond lengths ( & 0.005 \\
\hline R.m.s.d., bond angles $\left({ }^{\circ}\right)$ & 0.932 \\
\hline \multicolumn{2}{|l|}{ No. of non-H atoms } \\
\hline Protein atoms & 2109 \\
\hline Solvent atoms & 269 \\
\hline Heterogen atoms & 1 \\
\hline \multicolumn{2}{|l|}{$B$ factors $\left(\AA^{2}\right)$} \\
\hline All & 26.0 \\
\hline Protein & 25.1 \\
\hline Ligand $\left(\mathrm{Zn}^{2+}\right)$ & 12.9 \\
\hline Water & 32.8 \\
\hline
\end{tabular}

\subsection{ITC and data analysis}

ITC experiments were performed using a VP-ITC instrument (MicroCal) in PatE gel-filtration buffer at $20^{\circ} \mathrm{C}$. Both $\mathrm{PatE}^{\prime}$ and PatG-DUF ${ }_{d i}$ were dialyzed into freshly prepared $\mathrm{PatE}^{\prime}$ gel-filtration buffer for $2 \mathrm{~d}$ (changing to fresh buffer after the first day) at $4^{\circ} \mathrm{C}$ to ensure that the buffers for the experiment were matched. A cell solution of $40 \mu M$ PatG-DUF $d i$ and a syringe solution of $600 \mu M$ PatE' were prepared (using dialysis buffer to dilute the samples) and degassed at $18^{\circ} \mathrm{C}$ for $15 \mathrm{~min}$. The $\mathrm{PatE}^{\prime}$ solution was titrated into the PatG-DUF ${ }_{d i}$ solution as follows: an initial injection of $2 \mu$ followed by injections of $5 \mu \mathrm{l}$ at a speed of $0.5 \mu \mathrm{min}^{-1}$ with a delay of $4 \mathrm{~min}$ between injections. The syringe was stirred at $307 \mathrm{rev} \mathrm{min}^{-1}$ for the duration of the experiment. The raw data were processed using the MicroCal Origin software. The baseline was adjusted and the integrations were performed manually.

\subsection{NMR binding experiments}

NMR experiments were performed at $10^{\circ} \mathrm{C}$ in a Bruker DRX500 spectrometer equipped with a $5 \mathrm{~mm}$ TXIz probe. The instrument was run using the TopSpin software (Bruker). The sample for binding experiments consisted of $100 \mu M{ }^{15} \mathrm{~N} \mathrm{PatE}^{\prime}$ in $\mathrm{PatE}^{\prime}$ gel-filtration buffer supplemented with $5 \% \mathrm{D}_{2} \mathrm{O}$. and a ${ }^{1} \mathrm{H}-{ }^{15} \mathrm{~N}$ HSQC spectrum was recorded. Aliquots of $1.6 \mathrm{~m} M$ PatG-DUF ${ }_{d i}$ were added to final concentrations of 50, 100 and $200 \mu M$ and HSQCs were recorded after each addition. The spectra were overlaid using TopSpin to identify changes (if any) in the spectra subsequent to the addition of PatG-DUF $d i$. The ${ }^{1} \mathrm{H}-{ }^{15} \mathrm{~N}$ HSQC spectra were acquired with Watergate water suppression (Piotto et al., 1992) at $1024 \times 128$ points and a digital resolution of 9.8 and $19.0 \mathrm{~Hz}$ for the ${ }^{1} \mathrm{H}$ and ${ }^{15} \mathrm{~N}$ dimensions, respectively. The experiment was repeated using the modified ${ }^{15} \mathrm{~N}$ PatE' containing four heterocycles under the same conditions. This resulted in a poorer signal-to-noise ratio, and therefore the experiment was repeated using $250 \mu M$ modified ${ }^{15} \mathrm{~N} \mathrm{PatE}^{\prime}$ and aliquots of $2 \mathrm{~m} M$ PatG-DUF ${ }_{d i}$ were added to final concentrations of 125,250 and $500 \mu M$.

\section{Results}

\subsection{Crystal structure of PatG-DUF}

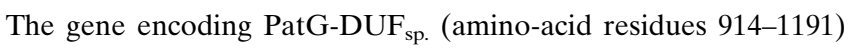
was amplified from gDNA isolated from Prochloron sp. Since we cannot be certain as to whether the variations between PatG-DUF $F_{\text {sp. }}$ and PatG-DUF ${ }_{d i}$ reflect natural variation amongst Prochloron species or have arisen from errors in the original amplification, we used site-directed mutagenesis to generate PatG-DUF with the sequence from $P$. didemni. Both PatG-DUF sp. $_{\text {(Glu958, Arg959 and }}$ Val1037) and PatG-DUF $d i$ (Asp958, Tyr959 and Met1037) were purified as described in $\$ 2$ and a near-UV CD spectrum revealed no significant difference (Supplementary Fig. S3). We concluded that either sequence was suitable for use in structural characterization. Crystallography was carried out on PatG-DUF $F_{\text {sp. }}$ as it had been purified first and gave crystals. We carried out biophysical studies with PatG-DUF $d i$ as we felt that these data would be more informative with a known database match.

The absence of PatG-DUF homologues in the PDB required the generation of a SeMet PatG-DUF $s p$. derivative, and the crystal structure was solved using single-wavelength anomalous dispersion. The SeMet PatG-DUF $F_{\text {sp. }}$ crystal belonged to space group $P 2_{1} 2_{1} 2$ and contained one monomer in the asymmetric unit. The structure has a fold comprising seven $\alpha$-helices and $13 \beta$-strands. Analysis with secondary-structure matching at the European Bioinformatics Institute (http://www.ebi.ac.uk/msd-srv/ssm/) and TopSearch (https:// topsearch.services.came.sbg.ac.at) both suggest that the fold is novel, with the closest matches being a series of unrelated enterotoxins giving r.m.s.d. values of between 3 and $4 \AA$ over $\sim 80$ residues (Krissinel \& Henrick, 2004; Wiederstein et al., 2014). The refined model contains residues 920-1064 and 1077-1184 (the residue numbers correspond to full-length PatG). The missing residues are at the $\mathrm{N}$ - and C-termini of the model and in a connecting loop between $\alpha$-helices 5 and 6 , and are presumed to be disordered. The model includes one disulfide bond between Cys1136 and Cys1142 and a single $\mathrm{Zn}^{2+}$ ion (Fig. 3a). Glu958 and Arg959 are located on the opposite face from the $\mathrm{Zn}^{2+}$ ion, whilst Val1037 is located on the edge of a hydrophobic pocket remote from the zinc (Fig. $3 a$ ). The changes in sequence would seem to be unlikely to alter the structure, which is consistent with the near-UV CD spectra. Since zinc was not deliberately added to any of the purification buffers or crystallization conditions, it is presumed the $\mathrm{Zn}^{2+}$ ion was bound during protein expression. The $\mathrm{Zn}^{2+}$ ion is coordinated in a tetrahedral arrangement by residues Glu977, His1087 and His1090. The fourth coordination site is occupied by residue Asp1088* from a symmetry mate (PatGDUF $F_{\mathrm{sp}}^{*}$ ), creating a dimer with two $\mathrm{Zn}^{2+}$-binding sites (Figs. $3 b$ and 3c). Analysis with PISA (http://www.ebi.ac.uk/pdbe/pisa/; Krissinel \& Henrick, 2007) reveals that there are almost no contacts between the protein residues, and removing the $\mathrm{Zn}^{2+}$ ions in silico reduces the complex formation significance score to 0.056 , indicating that $\mathrm{Zn}^{2+}$ is crucial in mediating dimer interactions. Since $\mathrm{Zn}^{2+}$ was not added, its presence in the crystal could suggest that the dimer is present in solution. However, PatG-DUF (both PatG-DUF sp. and PatG-DUF $_{d i}$ ) eluted from the gel-filtration column with a retention time consistent with a monomer (Fig. 4), preventing us from a firm assignment of dimer versus monomer in solution. Interestingly, the residues involved in $\mathrm{Zn}^{2+}$ binding are not all conserved in PatA-DUF (Fig. 1a). While the $\mathrm{H} 1087 \mathrm{Y}$ substitution might still coordinate $\mathrm{Zn}^{2+}$, the H1090R substitution would not. Additionally, these residues are not well conserved in the majority of the PatG-DUF homologues (with notable exceptions being TruG-DUF and ArtG-DUF) or in any of the PatA-DUF homologues (Supplementary Fig. S1). Consequently, we 


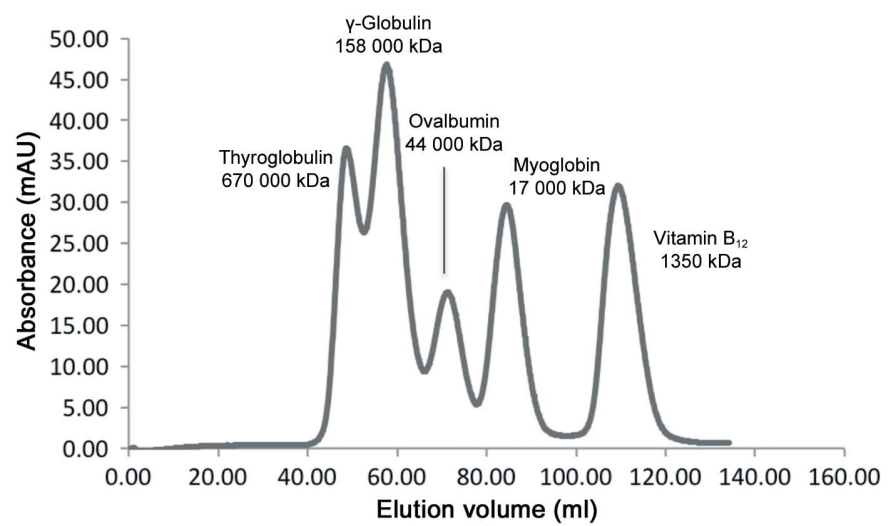

(a)

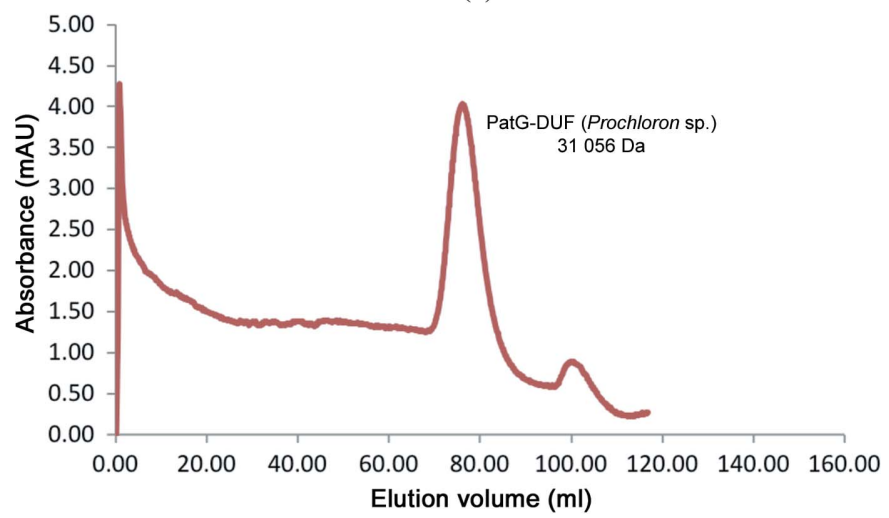

(b)

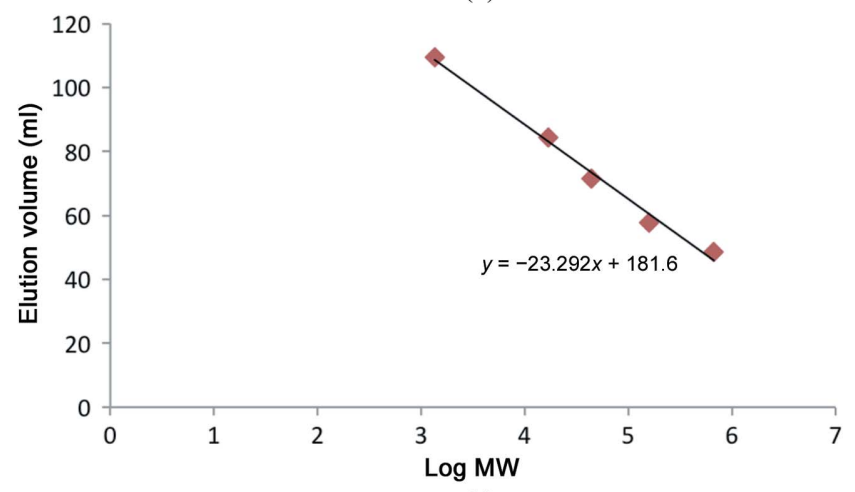

(c)

Figure 4

(a) Superdex 75 gel-filtration chromatogram of gel-filtration standards (Bio-Rad). (b) Superdex 75 gel-filtration chromatogram of PatG-DUF. (c) Standard curve (elution volume versus log MW). Interpolating from the standard curve, we can determine that PatG-DUF elutes from the gel-filtration column as a monomer.

cannot make any claims regarding the biological relevance of a dimeric assembly for the DUF domain.

The structure of PatG-DUF $F_{\text {sp. }}$ is novel and therefore provides no immediate insight into its function within patellamide biosynthesis. In an attempt to determine whether the C-terminal DUFs are directly involved in the biosynthesis, binding experiments between PatGDUF $_{d i}$ and the precursor peptide $\mathrm{PatE}^{\prime}$, and a modified precursor subsequent to heterocyclization by PatD, were performed using ITC and NMR.

\subsection{Investigating potential binding partners for PatG-DUF $d i$}

Titrating unmodified PatE' into a solution of PatG-DUF ${ }_{d i}$ reproducibly results in a binding curve (Supplementary Fig. S4). However,

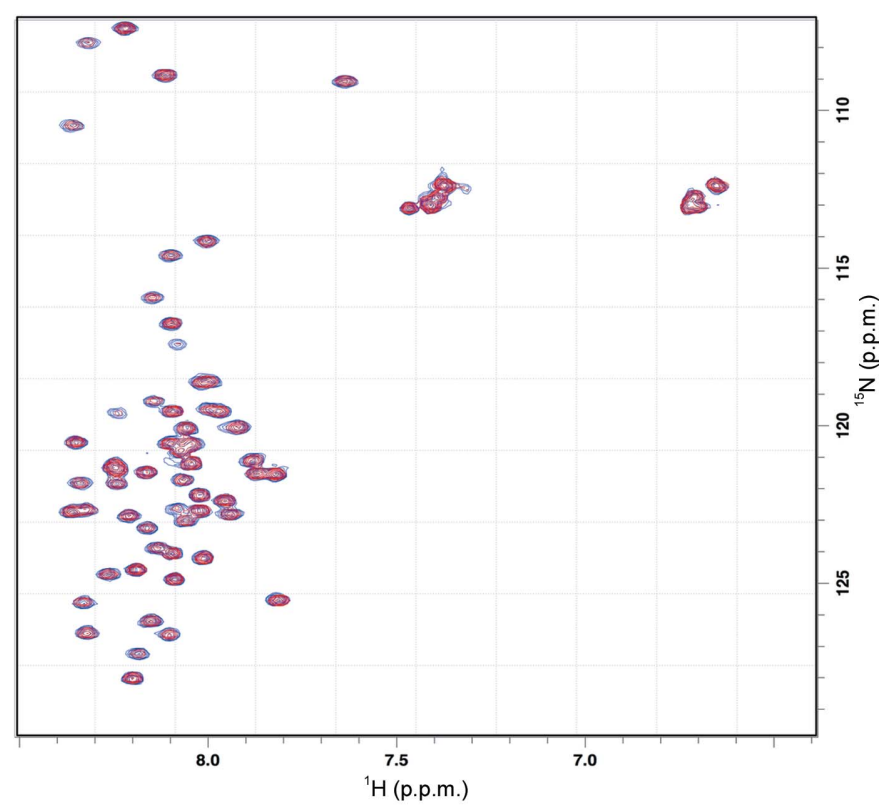

(a)

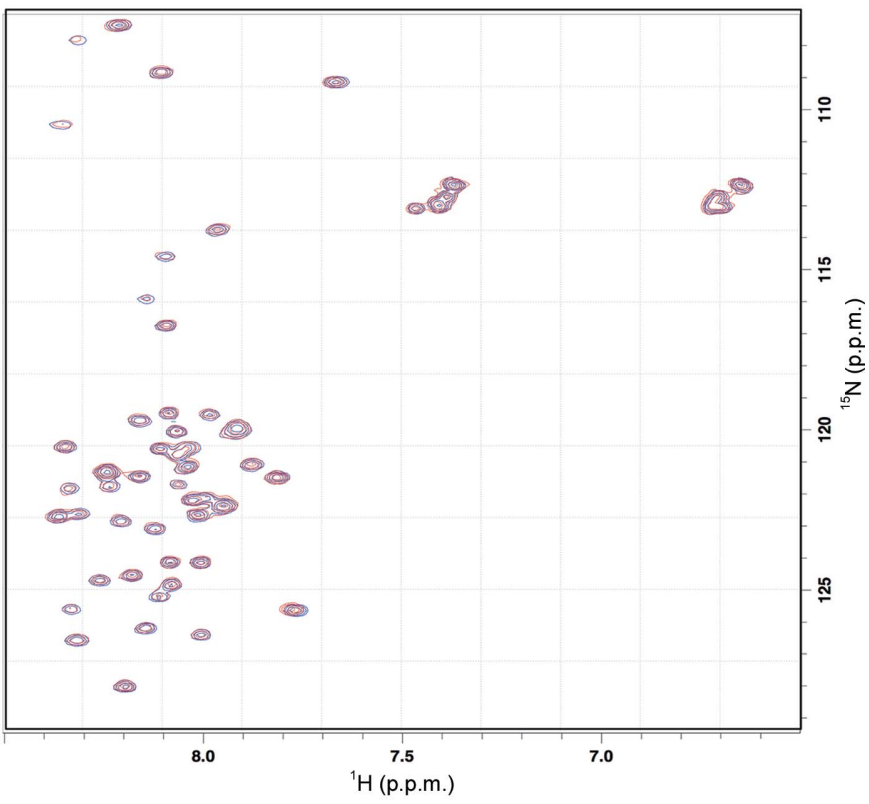

(b)

Figure 5

(a) Overlaid ${ }^{1} \mathrm{H}_{-}{ }^{15} \mathrm{~N}$ HSQC NMR spectra of PatE' before (blue) and after (red) the addition of 2.0 equivalents of PatG-DUF ${ }_{d i}(b)$ Overlaid ${ }^{1} \mathrm{H}-{ }^{15} \mathrm{~N}$ HSQC NMR spectra of heterocyclized PatE' before (blue) and after (red) the addition of 2.0 equivalents of PatG-DUF $d i$

analysis of the data yields unlikely stoichiometries of around ten molecules of PatG-DUF ${ }_{d i}$ binding to one molecule of PatE'. Close inspection of the cell revealed heavy precipitation at the end of the experiment, suggesting that we were measuring protein aggregation. Using ${ }^{15} \mathrm{~N}$-labelled PatE', a ${ }^{1} \mathrm{H}-{ }^{15} \mathrm{~N}$ HSQC spectrum was recorded in the absence and presence of $0.5,1.0$ and 2.0 molar equivalents of PatG-DUF $d i$ (Supplementary Fig. S5a). The spectra of pure ${ }^{15} \mathrm{~N} \mathrm{PatE}^{\prime}$ in the absence (blue) and presence (red) of 2.0 equivalents of PatGDUF $_{d i}$ have been overlaid (Fig. $5 a$ ), and clearly show an identical chemical shift pattern, showing that PatG-DUF ${ }_{d i}$ does not bind the unmodified precursor peptide. The NMR experiments were repeated using ${ }^{15} \mathrm{~N}$ PatE' that had been processed with PatD, introducing four heterocycles (two thiazolines and two oxazolines) within the context 
of the core peptide. As before, the addition of up to 2.0 equivalents of PatG-DUF $d i$ to the modified PatE' resulted in an identical NMR spectrum (Fig. $5 b$ and Supplementary Fig. S $5 b$ ), indicating that the heterocycle-containing peptide does not bind PatG-DUF ${ }_{d i}$ either. The known disorder of the leader peptide of PatE (Koehnke et al., 2013) would suggest that blocking of PatG-DUF fin $_{d i}$ binding by the leader peptide is unlikely. At present, we are unable to produce labelled cleaved precursor or macrocycle in sufficient quantities and purity for NMR experiments.

\section{Conclusions}

The patellamide proteins PatA and PatG contain C-terminal domains of unknown function which are $56 \%$ identical to each other and are conserved in related cyanobactin biosynthetic pathways. X-ray crystallography revealed the structure of PatG-DUF ${ }_{\text {sp. }}$ to be a novel fold and, although a dimer is observed in the crystal, the residues involved in $\mathrm{Zn}^{2+}$ coordination (which is required for dimerization) are not well conserved in PatA-DUF or in homologues from other pathways. Consequently, it is difficult to speculate as to the functional importance of the dimer. We have been unable to detect any binding to linear substrates (simple peptides or peptides with heterocycles), indicating that these are unlikely to be substrates or ligands of the DUFs. The pitfall in using one technique to assess binding was shown by the reproducible but functionally meaningless ITC binding curves that we observed. Further study is required with macrocycles before we can rule out any binding of the DUF domain to patellamide substrates. It remains possible that the dimerization of the domain indicates an interaction between the DUFs, potentially leading to homodimers of PatA/PatG or even heterodimers of PatA and PatG, which might be important for the activity of these enzymes in vivo. Further study is required to investigate such hypotheses. If the DUF domain has no enzymatic role, coupled with the observation that the protease domains of PatA and PatG are active in isolation, it is interesting as to why it is conserved throughout cyanobactin biosynthetic pathways. However, it also suggests that it could be excluded from in vitro enzyme-based synthesis systems. This simplifies the design and use of a biotechnological 'toolkit' for bioactive cyclic and linear peptides.

We thank the staff of beamline I02 at the Diamond Light Source for their support with data collection. Thanks go to the NMR and BSRC mass-spectrometry facilities at the University of St Andrews for crucial support. This work was funded by the BBSRC (BB/
K015508/1) and ERC (TNT-LEAP), and the University of St Andrews infrastructure is supported by a Wellcome Trust Capital Award (086036). WH is the recipient of the SULSA Leaders award.

\section{References}

Adams, P. D. et al. (2010). Acta Cryst. D66, 213-221.

Agarwal, V., Pierce, E., McIntosh, J., Schmidt, E. W. \& Nair, S. K. (2012). Chem. Biol. 19, 1411-1422.

Bent, A. F., Koehnke, J., Houssen, W. E., Smith, M. C. M., Jaspars, M. \& Naismith, J. H. (2013). Acta Cryst. F69, 618-623.

Bond, C. S. \& Schüttelkopf, A. W. (2009). Acta Cryst. D65, 510-512.

Chen, V. B., Arendall, W. B., Headd, J. J., Keedy, D. A., Immormino, R. M., Kapral, G. J., Murray, L. W., Richardson, J. S. \& Richardson, D. C. (2010). Acta Cryst. D66, 12-21.

DeLano, W. L. (2002). PyMOL. http://www.pymol.org.

Emsley, P. \& Cowtan, K. (2004). Acta Cryst. D60, 2126-2132.

Houssen, W. E. \& Jaspars, M. (2010). Chembiochem, 11, 1803-1815.

Houssen, W. E., Koehnke, J., Zollman, D., Vendome, J., Raab, A., Smith, M. C. M., Naismith, J. H. \& Jaspars, M. (2012). Chembiochem, 13, 26832689.

Jancarik, J. \& Kim, S.-H. (1991). J. Appl. Cryst. 24, 409-411.

Koehnke, J., Bent, A., Houssen, W. E., Zollman, D., Morawitz, F., Shirran, S., Vendome, J., Nneoyiegbe, A. F., Trembleau, L., Botting, C. H., Smith, M. C. M., Jaspars, M. \& Naismith, J. H. (2012). Nature Struct. Mol. Biol. 19, 767-772.

Koehnke, J., Bent, A. F., Zollman, D., Smith, K., Houssen, W. E., Zhu, X., Mann, G., Lebl, T., Scharff, R., Shirran, S., Botting, C. H., Jaspars, M., Schwarz-Linek, U. \& Naismith, J. H. (2013). Angew. Chem. Int. Ed. Engl. 52, 13991-13996

Krissinel, E. \& Henrick, K. (2004). Acta Cryst. D60, 2256-2268.

Krissinel, E. \& Henrick, K. (2007). J. Mol. Biol. 372, 774-797.

Liu, H. \& Naismith, J. H. (2008). BMC Biotechnol. 8, 91.

Liu, H. \& Naismith, J. H. (2009). Protein Expr. Purif. 63, 102-111.

Milne, B. F., Long, P. F., Starcevic, A., Hranueli, D. \& Jaspars, M. (2006). Org. Biomol. Chem. 4, 631-638.

Murshudov, G. N., Skubák, P., Lebedev, A. A., Pannu, N. S., Steiner, R. A., Nicholls, R. A., Winn, M. D., Long, F. \& Vagin, A. A. (2011). Acta Cryst. D67, 355-367.

Painter, J. \& Merritt, E. A. (2006). Acta Cryst. D62, 439-450.

Piotto, M., Saudek, V. \& Sklenář, V. (1992). J. Biomol. NMR, 2, 661-665.

Schmidt, E. W., Nelson, J. T., Rasko, D. A., Sudek, S., Eisen, J. A., Haygood, M. G. \& Ravel, J. (2005). Proc. Natl Acad. Sci. USA, 102, 7315-7320.

Sievers, F., Wilm, A., Dineen, D., Gibson, T. J., Karplus, K., Li, W., Lopez, R., McWilliam, H., Remmert, M., Söding, J., Thompson, J. D. \& Higgins, D. G. (2014). Mol. Syst. Biol. 7, 539.

Sivonen, K., Leikoski, N., Fewer, D. P. \& Jokela, J. (2010). Appl. Microbiol. Biotechnol. 86, 1213-1225.

Wiederstein, M., Gruber, M., Frank, K., Melo, F. \& Sippl, M. J. (2014). Structure, 22, 1063-1070.

Williams, A. B. \& Jacobs, R. S. (1993). Cancer Lett. 71, 97-102. 